\title{
Derivation of the Boltzmann Equation for Financial Brownian Motion: Direct Observation of the Collective Motion of High-Frequency Traders
}

\author{
Kiyoshi Kanazawa, ${ }^{1,2, *}$ Takumi Sueshige, ${ }^{2}$ Hideki Takayasu, ${ }^{1,3}$ and Misako Takayasu ${ }^{1,2}$ \\ ${ }^{1}$ Institute of Innovative Research, Tokyo Institute of Technology, 4259 Nagatsuta-cho, Midori-ku, Yokohama 226-8502, Japan \\ ${ }^{2}$ Department of Mathematical and Computing Science, School of Computing, \\ Tokyo Institute of Technology, 4259 Nagatsuta-cho, Midori-ku, Yokohama 226-8502, Japan \\ ${ }^{3}$ Sony Computer Science Laboratories, 3-14-13 Higashi-Gotanda, Shinagawa-ku, Tokyo 141-0022, Japan
}

(Received 10 May 2017; revised manuscript received 24 January 2018; published 27 March 2018)

\begin{abstract}
A microscopic model is established for financial Brownian motion from the direct observation of the dynamics of high-frequency traders (HFTs) in a foreign exchange market. Furthermore, a theoretical framework parallel to molecular kinetic theory is developed for the systematic description of the financial market from microscopic dynamics of HFTs. We report first on a microscopic empirical law of traders' trend-following behavior by tracking the trajectories of all individuals, which quantifies the collective motion of HFTs but has not been captured in conventional order-book models. We next introduce the corresponding microscopic model of HFTs and present its theoretical solution paralleling molecular kinetic theory: Boltzmann-like and Langevin-like equations are derived from the microscopic dynamics via the Bogoliubov-Born-Green-Kirkwood-Yvon hierarchy. Our model is the first microscopic model that has been directly validated through data analysis of the microscopic dynamics, exhibiting quantitative agreements with mesoscopic and macroscopic empirical results.
\end{abstract}

DOI: 10.1103/PhysRevLett.120.138301

Introduction.-In physics, the study of colloidal Brownian motion has a long history beginning with Einstein's famous work [1]; the understanding of its mechanism has been systematically developed in kinetic theory [2,3]. Specifically, from microscopic Newtonian dynamics, the Boltzmann and Langevin equations are derived for the mesoscopic and macroscopic dynamics, respectively. This framework is a rigid foundation for various nonequilibrium systems (e.g., active matter, granular gas, Feynman ratchets, and traffic flow [4-10]), and its direct experimental foundation has been revisited because of recent technological breakthroughs $[11,12]$.

In light of this success, it is natural to apply this framework beyond physics to social science [13], such as finance. Indeed, the concept of random walks was historically invented for price dynamics by Bachelier earlier than Einstein [14], and its similarities to physical Brownian motion (e.g., the fluctuation-dissipation relation) are intensively studied by recent high-frequency data analysis [15]. As an idea in statistical physics, the dynamics of financial markets are expected to be clarified from first principles by extending kinetic theory.

Published by the American Physical Society under the terms of the Creative Commons Attribution 4.0 International license. Further distribution of this work must maintain attribution to the author(s) and the published article's title, journal citation, and DOI.
Although this idea is attractive, the kinetic description has not been established for financial Brownian motion. Why has not this idea been realized yet? In our view, the biggest problem is the absence of established microscopic models; there exist empirical validations of mesoscopic [15-21] and macroscopic models [22-28], whereas no microscopic model has been validated by direct empirical analysis. Indeed, previous microscopic models [29-33] were purely theoretical and have no quantitative evidence microscopically. To overcome this crucial problem as an empirical science, two missing links have to be connected: (i) establishment of the microscopic model by direct observation of traders' dynamics [Fig. 1(a)] and (ii) construction of a kinetic theory to show its consistency with mesoscopic and macroscopic findings [i.e., the order-book and price dynamics, Figs. 1(b), 1(c)].

In this Letter, we present the corresponding solutions by direct observation of high-frequency trader (HFT) dynamics in a foreign exchange (FX) market: (i) a microscopic model of HFTs is established by direct microscopic evidence, and (ii) corresponding kinetic theory is developed to show its consistency with mesoscopic and macroscopic evidence. We analyzed order-book data with anonymized trader identifiers (IDs) to track trajectories of all individuals. We found an empirical law concerning trend following among HFTs, which has not been captured by previous order-book models. Remarkably, this property induces the collective motion of the order book and naturally leads the layered order-book structure [15]. We then introduce a 

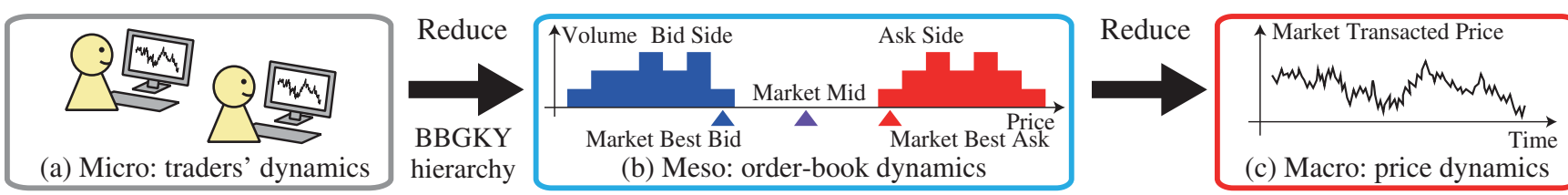

FIG. 1. Hierarchies for financial Brownian motions for the microscopic, mesoscopic, and macroscopic dynamics.

corresponding microscopic model of trend-following HFTs. Starting from their "equations of motion," Boltzmann-like and Langevin-like equations are derived for the order-book and price dynamics. A quantitative agreement is finally shown with our empirical all findings. Our work opens the door to systematic descriptions of finance based on microscopic evidence.

Observed microscopic dynamics.-We analyzed the high-frequency FX data between the U.S. dollar (USD) and the Japanese Yen (JPY) on Electronic Broking Services for a week in June 2016. The currency unit used in this study is 0.001 yen, called the tenth pip (tpip). Here we particularly focused on the dynamics of HFTs [34], frequently submitting or canceling orders according to algorithms (see Supplemental Material [35]). The typical trajectories of bid and ask quoted prices are illustrated in Figs. 2(a)-2(c) for the top 3 HFTs. They modify their quoted prices by successive submission and cancellation at high speed typically within seconds; almost $99 \%$ of their submissions were finally canceled without transactions (see Supplemental Material [35]). With the two-sided quotes they also play the role of liquidity providers [40,41] according to the market rule, keeping the balance between the bid and ask order book. Buy-sell spreads, the difference between the best bid and ask prices for a single HFT, were observed to fluctuate around certain time constants (see the insets for their distributions).

We then report the empirical microscopic law for the trend-following strategy of individual traders. The bid and ask quoted prices of the top $i$ th HFT are denoted by $b_{i}$ and $a_{i}$ (see Supplemental Material [35]). We investigated the average movement of the trader's quoted midprice $z_{i} \equiv$ $\left(b_{i}+a_{i}\right) / 2$ between transactions conditional on the previous market transacted price movement [Fig. 2(d)]. Here we introduce the tick time $T$ as an integer time incremented by every transaction. The mean transaction interval is $9.3 \mathrm{sec}$ during this week. Because typical HFTs frequently modify their price between transactions, we here study HFTs' trend following at one-tick precision. For the top 20 HFTs (Fig. 3), we found that the average and variance of movement $\Delta z_{i}(T) \equiv z_{i}(T+1)-z_{i}(T)$ obeyed

$$
\left\langle\Delta z_{i}\right\rangle_{\Delta p} \approx c_{i} \tanh \frac{\Delta p}{\Delta p_{i}^{*}}, \quad V_{\Delta p}\left[\Delta z_{i}\right] \approx \sigma_{i}^{2}
$$

where the conditional average $\langle\ldots\rangle_{\Delta p}$ is taken when the last price change is $\Delta p(T-1) \equiv p(T)-p(T-1)$ and $\Delta z_{i} \neq 0$ (see Supplemental Material [35]) and the conditional variance is defined by $V_{\Delta p}\left[\Delta z_{i}\right] \equiv\left\langle\left(\Delta z_{i}-\left\langle\Delta z_{i}\right\rangle_{\Delta p}\right)^{2}\right\rangle_{\Delta p}$. Here, $p(T)$ is the market transacted price at the $T$ tick, and $c_{i}, \Delta p_{i}^{*}, \sigma_{i}^{2}$ are characteristic constants unique to the trader and independent of $\Delta p$. Their typical values were found to be $c_{i} \approx 6.0$ tpip, $\Delta p_{i}^{*} \approx 7.5$ tpip, and $\sigma_{i} \approx 14.5$ tpip. Our finding (1) implies that the reaction of traders is linear for small trends but saturates for large trends, and quantifies the collective motion of HFTs. Remarkably, a similar behavior was reported from a price movement data analysis at onemonth precision [42].

Microscopic model.-Here we introduce a minimal microscopic model of HFTs incorporating the above characters. We make four assumptions: (i) The number of traders is sufficiently large; (ii) traders always quote both bid and ask prices (for the $i$ th trader, $b_{i}$ and $a_{i}$ ) simultaneously with a unit volume; (iii) buy-sell spreads are time constants unique to traders with distribution $\rho(L)$. The trader dynamics are then characterized by the midprice $z_{i} \equiv\left(b_{i}+a_{i}\right) / 2$; and (iv) trend-following random walks are assumed in the microscopic dynamics [Figs. 4(a)-4(c)],

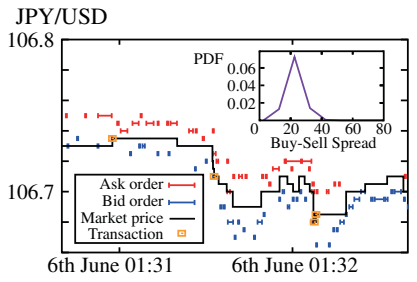

(a) 1 st top HFT

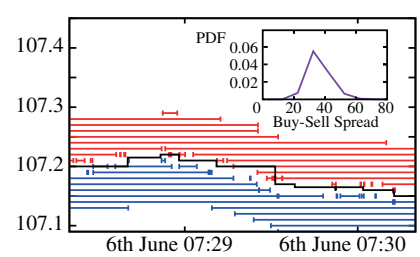

(b) 2nd top HFT

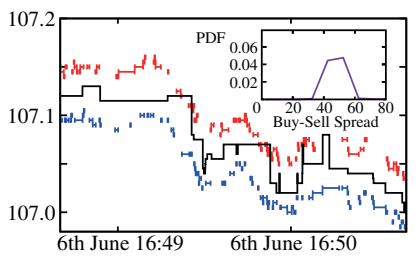

(c) 3rd top HFT

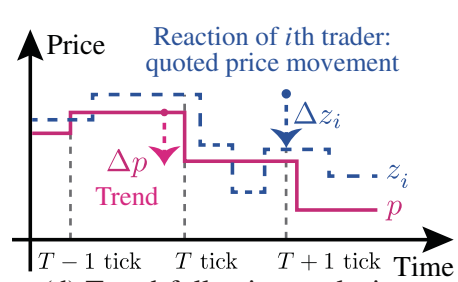

(d) Trend-following analysis

FIG. 2. (a)-(c) Lifetimes of orders are plotted as trajectories for the top 3 HFTs. Typical traders tend towards continuous two-sided quotes, with the buy-sell spread fluctuating around a time constant unique to the trader. The percentage of two-sided quotes among HFTs was $48.4 \%$ (see Supplemental Material [35]). (d) Quantification of trend following for individual traders, where $\Delta p$ and $\Delta z_{i}$ are the movements of the market price and the midprice of the $i$ th trader, respectively. 


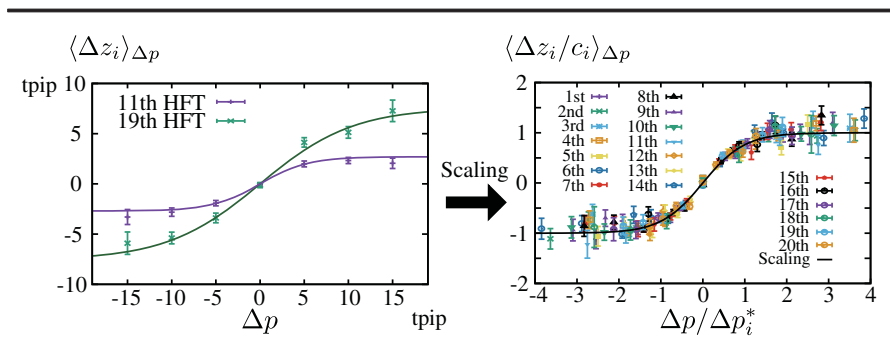

(a) Trend-following movement on average (hyperbolic function)

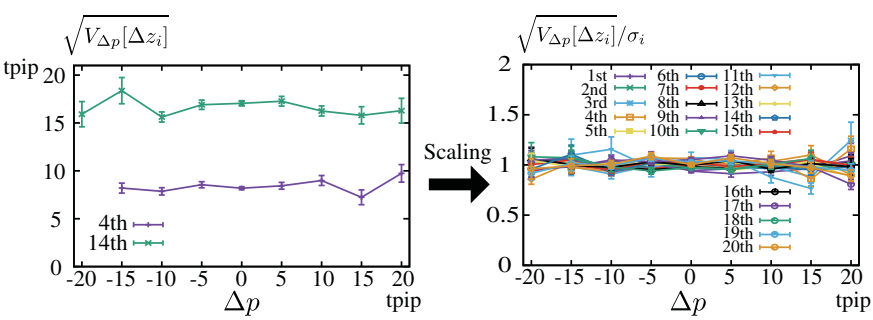

(b) Standard deviation of random noise effect (independent of $\Delta p$ )

FIG. 3. (a) Average $\Delta z_{i}$ assuming previous price movements of $\Delta p$ and an active trader with $\Delta z_{i} \neq 0$. The behavior can be fitted by the master curve (1) for the top 20 HFTs by introducing scaling parameters $\Delta p_{i}^{*}$ and $c_{i}$. (b) Conditional standard deviation on price movement $\Delta p$, showing that the randomness associated with trend following is independent of $\Delta p$.

$$
\frac{d z_{i}(t)}{d t}=c \tanh \frac{\Delta p(t)}{\Delta p^{*}}+\sigma \eta_{i}^{R}(t)
$$

with strength for trend following $c$, previous price movement $\Delta p$, and white Gaussian noise $\sigma \eta_{i}^{R}$ with variance $\sigma^{2}$. Here, $c, \Delta p^{*}$, and $\sigma$ are assumed shared for all traders for simplicity. In this model, HFTs frequently modify their quoted price by successive submission and cancellation. Indeed, this model can be reformulated as a Poisson price modification process with high cancellation rate (see Supplemental Material [35]). After transaction $a_{j}(t)=$ $b_{i}(t)$ [Fig. 4(b)], the updated market price and its corresponding movement are recorded as

$$
p(t+0)=b_{i}(t), \quad \Delta p(t+0)=b_{i}(t)-p(t),
$$

and a requotation jump occurs [Fig. 4(c)],

$z_{i}(t+0)=z_{i}(t)-\frac{L_{i}}{2}, \quad z_{j}(t+0)=z_{j}(t)+\frac{L_{j}}{2}$.

Here, $t+0$ implies the time after transaction. A unique character of this model is the order-book collective motion due to trend following [Fig. 4(d)]. For $\Delta p>0$, the bid (ask) volume change tends to be positive (negative) near the best price [Fig. 4(e)], consistently with the layered order-book structure [15].
Kinetic formulation.-We next present an analytical solution to this model (2) according to kinetic theory $[2,3]$. Let us first introduce the relative distance $r_{i} \equiv z_{i}-z_{\text {c.m. }}$. from the "center of mass" $z_{\text {c.m. }} \equiv \sum_{i} z_{i} / N$ [Fig. 4(a)], where the trend-following effect in Eq. (2) is absorbed into the dynamics of $z_{\text {c.m. }}$. The dynamics of $r_{i}$ become simpler because trend-following effects disappear in this moving frame (see Supplemental Material [35]). We next introduce the one-body (two-body) probability distribution as $\phi_{L}(r)$ $\left[\phi_{L L^{\prime}}\left(r, r^{\prime}\right)\right]$ conditional on traders' buy-sell spreads. From the microscopic model (2), the lowest-order hierarchy equation is derived as $\partial \phi_{L} / \partial t=\left(\sigma^{2} / 2\right)\left(\partial^{2} \phi_{L} / \partial r^{2}\right)+$ $N \sum_{s= \pm 1} \int d L^{\prime} \rho\left(L^{\prime}\right)\left[J_{L L^{\prime}}^{s}(r+s L / 2)-J_{L L^{\prime}}^{s}\right]$ with $J_{L L^{\prime}}^{s}(r) \equiv$ $\left.\left(\sigma^{2} / 2\right)\left|\tilde{\partial}_{r r^{\prime}}\right| \phi_{L L^{\prime}}\left(r, r^{\prime}\right)\right|_{r-r^{\prime}=s\left(L+L^{\prime}\right) / 2}$ and $\left|\tilde{\partial}_{r r^{\prime}}\right| f \equiv|\partial f / \partial r|+$ $\left|\partial f / \partial r^{\prime}\right|$ (see Supplemental Material [35]). By assuming "molecular chaos,"

$$
\phi_{L L^{\prime}}\left(r, r^{\prime}\right) \approx \phi_{L}(r) \phi_{L^{\prime}}\left(r^{\prime}\right),
$$

we derive the Boltzmann-like equation with collision integrals for the order book,

$$
\begin{aligned}
\frac{\partial \phi_{L}}{\partial t} \approx & \frac{\sigma^{2}}{2} \frac{\partial^{2} \phi_{L}}{\partial r^{2}} \\
& +N \sum_{s= \pm 1} \int d L^{\prime} \rho\left(L^{\prime}\right)\left[\tilde{J}_{L L^{\prime}}^{s}(r+s L / 2)-\tilde{J}_{L L^{\prime}}^{s}\right]
\end{aligned}
$$

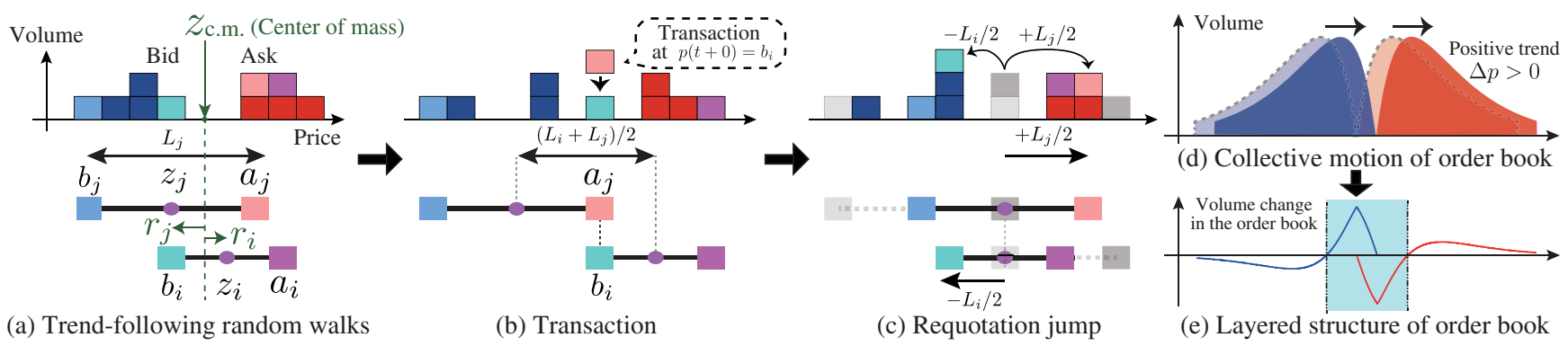

FIG. 4. Schematic of the microscopic model (2). (a) Midprice of each trader obeys trend-following random walks. (b) Transaction takes place after price matching $b_{i}=a_{j}$ with market transacted price $p$ and its movement $\Delta p$ updated. (c) A pair of traders requote their bid and ask prices simultaneously after transactions. (d) Order-book collective motion induced by trend following. (e) Volume change in the bid (ask) order book is positive (negative) near the best price on average for $\Delta p>0$. 
with $\left.\quad \tilde{J}_{L L^{\prime}}^{s}(r) \equiv\left(\sigma^{2} / 2\right)\left|\tilde{\partial}_{r r^{\prime}}\right|\left\{\phi_{L}(r) \phi_{L^{\prime}}\left(r^{\prime}\right)\right\}\right|_{r-r^{\prime}=s\left(L+L^{\prime}\right) / 2}$. Here, $s=+1(s=-1)$ represents transactions as bidder (asker). Because traders exhibit collective motion arising from trend following, a Langevin-like equation is also derived as the macroscopic description of the model (2),

$$
\Delta p(T+1)=c \tau(T) \tanh \frac{\Delta p(T)}{\Delta p^{*}}+\zeta(T),
$$

where $\tau(T)$ and $\zeta(T)$ are transaction time interval and random noise at the $T$ th tick time, respectively. The first trend-following term corresponds to the momentum inertia in the conventional Langevin equation.

Equations (6) and (7) can be analytically assessed for $N \rightarrow \infty$. We first set the buy-sell spread distribution as

$$
\rho(L)=\frac{L^{3}}{6 L^{* 4}} e^{-L / L^{*}},
$$

with decay length $L^{*}=15.5 \pm 0.2$ tpip, empirically validated in our data set [Fig. 5(a) and Supplemental Material [35]]. The solution to Eq. (6) for $N \rightarrow \infty$ is given by $\phi_{L}(r)=\left(4 / L^{2}\right) \max \{L / 2-|r|, 0\}$. The average orderbook profile $f_{\mathrm{A}}(r)=\int d L \rho(L) \phi_{L}(r-L / 2)$ is then given for $r>0$ by

$$
f_{\mathrm{A}}(r)=\frac{4 e^{-\frac{3 r}{2 L^{*}}}}{3 L^{*}}\left[\left(2+\frac{r}{L^{*}}\right) \sinh \frac{r}{2 L^{*}}-\frac{r e^{-\frac{r}{2 L^{*}}}}{2 L^{*}}\right] .
$$

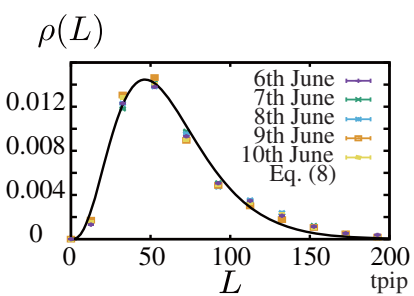

(a) Daily buy-sell spread dist.

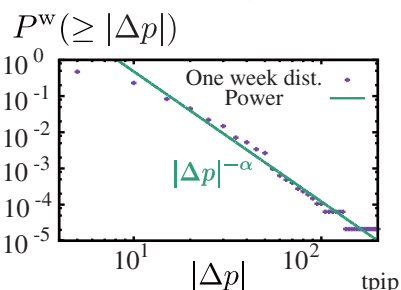

(e) One week price movement CDF

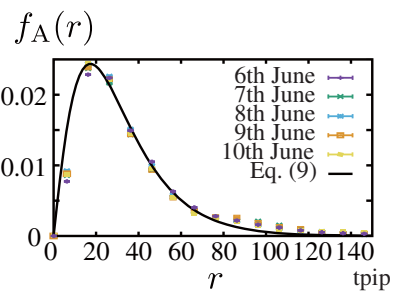

(b) Daily order-book profile

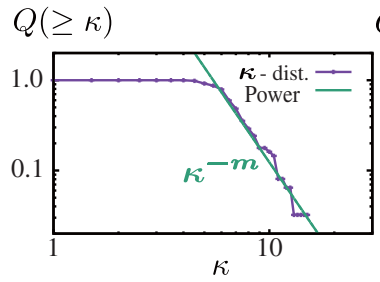

(f) Decay length CDF

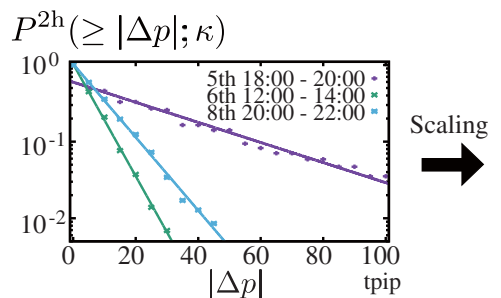

(c) Price movement CDF (one tick)

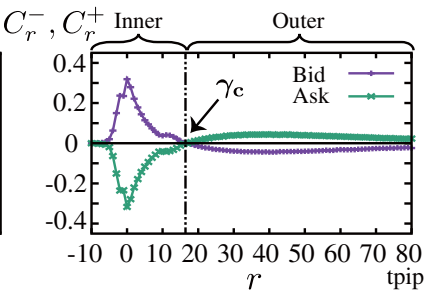

(g) Layered structure of order book
The statistics of $\tau(T)$ in the macroscopic model (7) is derived from the mesoscopic model (6), and the tail of the price movement is approximately given by

$$
P(\geq|\Delta p| ; \kappa) \approx e^{-|\Delta p| / \kappa} \quad(|\Delta p| \rightarrow \infty),
$$

with decay length $\kappa \approx 2 \Delta z^{*} / 3$, average movement from trend-following $\Delta z^{*} \equiv c \tau^{*}$, average transaction interval $\tau^{*} \approx 3 L^{* 2} / N \sigma^{2}$, and complementary cumulative distribution function (CDF) $P(\geq|\Delta p| ; \kappa)$ (see also Supplemental Material [35] for numerical validation).

Mesoscopic and macroscopic data analysis.-We next investigated whether our microscopic model is consistent with our data set. The empirical daily profile was first studied for the average ask order book for the best prices of HFTs $f_{\mathrm{A}}(r)$ [Fig. 5(b) and Supplemental Material [35]]. Surprisingly, we found a quantitative agreement with our theory (9) without any fitting parameters, which strongly supports the validity of our description.

The two-hourly segmented CDF for the price movement is also evaluated in one-tick precision $P^{2 \mathrm{~h}}(\geq|\Delta p| ; \kappa)$ [Fig. 5(c)], which obeys an exponential law that is qualitatively consistent with our theoretical prediction (10). The value of the two-hourly decay length $\kappa$ fluctuates significantly during a week. To remove this nonstationary feature, we introduced the two-hourly scaled CDF $\tilde{P}^{2 \mathrm{~h}}(\geq|\Delta \tilde{p}|) \equiv P^{2 \mathrm{~h}}(\geq \kappa|\Delta \tilde{p}| ; \kappa) / Z$ with scaling parameters $\kappa$ and $Z$ [Fig. 5(d)], thereby incorporating the two-hourly exponential law for the whole week.

FIG. 5. (a) Daily distribution of buy-sell spreads for HFTs with the empirical master curve (8). (b) Daily average order-book profile for the best prices of HFTs, agreeing with our theoretical line (9) without fitting parameters. Here the relative depth is measured from the market midprice instead of the c.m. for simplicity, which did not cause big numerical difference in comparing with our theory (see Supplemental Material [35]). (c) Two-hourly segmented CDFs for the price movement in one-tick precision for the three typical time regions (see Supplemental Material [35]). The CDFs are exponential, consistently with our theoretical prediction (10). (d) Two-hourly segmented CDFs are scaled into the single exponential master curve every 2 hours (62 time regions). (e) Price movement CDF over the whole week obeys a power-law of exponent $\alpha$. (f) Decay length $\kappa$ obeys a power-law $Q(\geq \kappa) \sim \kappa^{-m}$. (g) Order-book layered structure by our HFT model. Pearson's coefficient $C_{r}^{-}\left(C_{r}^{+}\right)$are numerically plotted between $N_{r}^{-}\left(N_{r}^{+}\right)$and $\Delta p$ with crossover point $\gamma_{\mathrm{c}} \approx 16.5$ tpip. (h) Linear correlation between the total number change $N_{\text {inner }}$ in the inner layer and the price movement $\Delta p$ with correlation coefficient of 0.63 . 
The price movements obey an exponential law for short periods but simultaneously obey a power law over long periods with exponent $\alpha=3.6 \pm 0.13$ [Fig. 5(e)]. This apparent discrepancy originates from the power-law nature of the decay length $\kappa$. Because $\kappa$ approximately obeys a power-law $\operatorname{CDF} Q(\geq \kappa) \sim \kappa^{-m}$ over the week with $m=$ $3.5 \pm 0.13$ [Fig. 5(f)], the one-week CDF $P^{\mathrm{w}}(\geq|\Delta p|)$ asymptotically obeys the power law as a superposition of the two-hourly segmented exponential CDF,

$$
P^{\mathrm{w}}(\geq|\Delta p|)=\int_{0}^{\infty} d \kappa Q(\kappa) P^{2 \mathrm{~h}}(\geq|\Delta p| ; \kappa) \propto|\Delta p|^{-m}
$$

with $Q(\kappa) \equiv-d Q(\geq \kappa) / d \kappa$, consistently with empirical exponent $\alpha \approx m$. Our result is therefore consistent with the previous reported power law [24-27] as a nonstationary property of $\kappa$.

Since our trend-following HFT model exhibits the orderbook collective motion [Figs. 4(d) and 4(e)], this model can reproduce the layered order-book structure [15] (see Supplemental Material [35]). Let us define $c_{r}^{-}\left(c_{r}^{+}\right)$and $a_{r}^{-}\left(a_{r}^{+}\right)$as the number of bid (ask) submissions and cancellations between one tick at the relative distance $r$ from the market midprice. We also define the number change $N_{r}^{-}=c_{r}^{-}-a_{r}^{-}\left(N_{r}^{+}=c_{r}^{+}-a_{r}^{+}\right)$at the distance $r$ for the bid (ask) side. Correlation coefficient $C_{r}^{-}\left(C_{r}^{+}\right)$is plotted in Fig. 5(g) between $N_{r}^{-}\left(N_{r}^{+}\right)$and $\Delta p$, showing positive and negative correlation in the inner (outer) and outer (inner) layers, respectively. We further show a linear correlation between the price movement $\Delta p$ and the total number change in the inner layer $N_{\text {inner }} \equiv$ $\int_{-\infty}^{\gamma_{c}} d r\left(N_{r}^{-}-N_{r}^{+}\right)$. The trend-following HFT model is thus qualitatively consistent with the previous findings [15] (see also Supplemental Material for data analyses [35]), implying that the layered structure was the direct consequence of the collective motion.

Discussion.-We have empirically studied the trend following of HFTs, inducing the collective motion of the order book. This property has not been captured in the previous order-book model [16-21] and was critical in reproducing our empirical findings. Indeed, none of our empirical findings, the order-book profile, the exponential price movement, and the layered order-book structure [15] were reproduced by the previous order-book model under realistic parameters in the absence of the collective motion (see Supplemental Material [35]). We expect that introduction of this collective motion to order-book models would be the key to replicate these empirical findings.

Conclusion.-We have established both a microscopic model and a kinetic theory for FX traders by direct observation of the HFTs' dynamics, quantitatively agreeing with empirical results under minimal assumptions. In the stream of econophysics, our model (2) is the first microscopic model directly supported by microscopic dynamical evidence and exhibiting agreement with mesoscopic and macroscopic findings. We expect that a new stream arises toward systematic descriptions of the financial market based on microscopic evidence. Interested readers are referred to Ref. [43] for more mathematical details.

We greatly appreciate NEX for their provision of the EBS data. We also appreciate M. Katori, H. Hayakawa, S. Ichiki, K. Yamada, S. Ogawa, F. van Wijland, D. Sornette, M. Sano, T. G. Sano, and T. Ito for fruitful discussions. This work was supported by JSPS KAKENHI (Grants No. 16K16016 and No. 17J10781) and JST, Strategic International Collaborative Research Program (SICORP) on the topic of "ICT for a Resilient Society" by Japan and Israel. We thank Richard Haase, Ph.D., from Edanz Group for editing a draft of this manuscript.

* Corresponding author.

kanazawa.k.ae@m.titech.ac.jp

[1] A. Einstein, Ann. Phys. (Berlin) 322, 549 (1905).

[2] S. Chapman and T. G. Cowling, The Mathematical Theory of Non-Uniform Gases (Cambridge University Press, Cambridge, England, 1970).

[3] N. G. van Kampen, Stochastic Processes in Physics and Chemistry, 3rd ed. (Elsevier, Amsterdam, 2007).

[4] E. Bertin, M. Droz, and G. Grégoire, Phys. Rev. E 74, 022101 (2006); J. Phys. A 42, 445001 (2009).

[5] N. V. Brilliantov and T. Pöschel, Kinetic Theory of Granular Gases (Oxford University Press, New York, 2004).

[6] C. Van den Broeck, R. Kawai, and P. Meurs, Phys. Rev. Lett. 93, 090601 (2004).

[7] C. Van den Broeck and R. Kawai, Phys. Rev. Lett. 96, 210601 (2006).

[8] D. Helbing, Rev. Mod. Phys. 73, 1067 (2001).

[9] A. Schadschneider, D. Chowdhury, and K. Nishinari, Stochastic Transport in Complex Systems: From Molecules to Vehicles (Elsevier, Amsterdam, 2010).

[10] I. Prigogine and R. Herman, Kinetic Theory of Vehicular Traffic (American Elsevier, New York, 1971).

[11] R. Huang, I. Chavez, K. M. Taute, B. Lukić, S. Jeney, M. G. Raizen, and E.-L.Florin, Nat. Phys. 7, 576 (2011).

[12] T. Li, S. Kheifets, D. Medellin, and M. G. Raizen, Science 328, 1673 (2010).

[13] L. Pareschi and G. Toscani, Interacting Multiagent Systems: Kinetic Equations and Monte Carlo Methods (Oxford University Press, Oxford, 2013).

[14] L. Bachelier, Ann. Sci. Éc. Norm. Supér. 17, 21 (1900).

[15] Y. Yura, H. Takayasu, D. Sornette, and M. Takayasu, Phys. Rev. Lett. 112, 098703 (2014); Phys. Rev. E 92, 042811 (2015).

[16] F. Slanina, Essentials of Econophysics Modelling (Oxford University Press, Oxford, 2014).

[17] S. Maslov, Physica (Amsterdam) 278A, 571 (2000).

[18] M. G. Daniels, J. D. Farmer, L. Gillemot, G. Iori, and E. Smith, Phys. Rev. Lett. 90, 108102 (2003).

[19] E. Smith, J. D. Farmer, L. Gillemot, and S. Krishnamurthy, Quant. Finance 3, 481 (2003). 
[20] J.-P. Bouchaud, M. Mézard, and M. Potters, Quant. Finance 2, 251 (2002).

[21] J. D. Farmer, P. Patelli, and I. Zovko, Proc. Natl. Acad. Sci. U.S.A. 102, 2254 (2005).

[22] R. N. Mantegna and H. E. Stanley, Nature (London) 376, 46 (1995).

[23] R. N. Mantegna and H. E. Stanley, An Introduction to Econophysics (Cambridge University Press, Cambridge, England, 1999).

[24] T. Lux, Appl. Financ. Econ. 6, 463 (1996).

[25] V. Plerou, P. Gopikrishnan, L. A. Nunes Amaral, M. Meyer, and H. E. Stanley, Phys. Rev. E 60, 6519 (1999).

[26] D. M. Guillaume, M. M. Dacorogna, R. R. Davé, U. A. Müller, R. B. Olsen, and O. V. Pictet, Finance Stoch. 1, 95 (1997).

[27] F. M. Longin, J. Business 69, 383 (1996).

[28] M. Takayasu, T. Mizuno, and H. Takayasu, Physica (Amsterdam) 370A, 91 (2006).

[29] A. S. Kyle, Econometrica 53, 1315 (1985).

[30] H. Takayasu, H. Miura, T. Hirabayashi, and K. Hamada, Physica (Amsterdam) 184A, 127 (1992).

[31] P. Bak, M. Paczuski, and M. Shubik, Physica (Amsterdam) 246A, 430 (1997).

[32] T. Lux and M. Marchesi, Nature (London) 397, 498 (1999).

[33] K. Yamada, H. Takayasu, T. Ito, and M. Takayasu, Phys. Rev. E 79, 051120 (2009).
[34] T. Hendershott, Ch. M. Jones, and A. J. Menkveld, J. Finance 66, 1 (2011).

[35] See Supplemental Material at http://link.aps.org/ supplemental/10.1103/PhysRevLett.120.138301 for the details of the data and theoretical analyses, which includes Refs. [36-39].

[36] A. B. Schmidt, Ecology of the Modern Institutional Spot FX: The EBS Market in 2011, SSRN 1984070 (2011).

[37] C. W. Gardiner, Handbook of Stochastic Methods for Physics, 4th ed. (Springer-Verlag, Berlin, 2009).

[38] E. A. Novikov, Sov. Phys. JETP 20, 1290 (1965); W. Horsthemke and R. Lefever, Noise-Induced Transitions: Theory and Applications in Physics, Chemistry, and Biology (Springer-Verlag, Berlin, 1984); R. F. Fox, Phys. Rev. A 33, 467 (1986).

[39] H. A. David and H. N. Nagaraja, Order Statistics, 3rd ed. (Wiley, New York, 2003).

[40] A. J. Menkveld, J. Financ. Mark. 16, 712 (2013); Annu. Rev. Financ. Econ. 8, 1 (2016).

[41] EBS Dealing Rules-Appendix-EBS Market (at the time of June 2016).

[42] Y. Lempérière, C. Deremble, P. Seager, M. Potters, and J.-P. Bouchaud, J. Investment Strategies. 3, 41 (2014).

[43] K. Kanazawa, T. Sueshige, H. Takayasu, and M. Takayasu, arXiv:1802.05993. 